\title{
Is electrocardiography sufficient for supraventricular tachycardia treatment? A case report
}

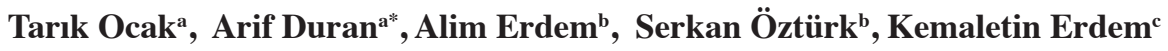 \\ ${ }^{a}$ Department of Emergency Medicine, Faculty of Medicine, Abant Izzet Baysal University, Bolu, Turkey \\ ${ }^{b}$ Department of Cardiology, Faculty of Medicine, Abant Izzet Baysal University, Bolu, Turkey \\ ${ }^{c}$ Department of Cardiovascular Surgery, Faculty of Medicine, Abant Izzet Baysal University, Bolu, Turkey
}

\section{ARTICLE INFO}

\section{Article History}

Received 15/01/2013

Accepted 28/03/2013

\section{* Correspondence to:}

Arif Duran

Department of Emergency Medicine,

Faculty of Medicine,

Abant Izzet Baysal University,

Bolu, Turkey

e-mail: drarifduran@gmail.com

\section{ABSTRACT}

In Wolff-Parkinson-White Syndrome (WPW), presence of accessory conduits causes various signs and clinical conditions in patients. WPW syndrome is presently defined as a congenital defect involving the occurrence of abnormal conductive tissue between the atria and the ventricles in relation to supraventricular tachycardia (SVT). SVT is observed in this group of patients. WPW syndrome at high rates can be mixed with SVT. Calcium channel blockers (diltiazem, verapamil) that are one of the most preferred medicines for rate control in patients with SVT may lead to fatal risks in individuals with WPW syndrome. In our presentation, we studied, in parallel to the literature, the case of a patient who presents the WPW syndrome but who is followed as a SVT without performing the electrophysiological tests in parallel to the literature.

J. Exp. Clin. Med., 2013; 30:357-359

\section{Keywords:}

Emergency medicine

Diltiazem

Supraventricular tachycardia

Wolf parkinson white syndrome

(C) 2013 OMU

\section{Introduction}

Wolf Parkinson White (WPW) syndrome is a clinical manifestation characterized by the presence of an electrical signing accessory pathway among atrium and the ventricle that is sometimes caused by tachyarrhythmia's may also cause sudden cardiac death (SCD). Previous studies have demonstrated that SCD occurs at the rate of $0.15 \%$ per year in WPW patients. These deaths are due to ventricular fibrillation developing as a result of rapid ventricular response of atrial fibrillation. Sometimes these patients wrongly use calcium channel blocker or beta blocker also these drugs increase ventricular response and leads to ventricular fibrillation. We present a WPW syndrome case that presented to the emergency service with narrow QRS tachycardia and interestingly using the diltiazem for two years.

\section{Case}

It has been determined by examination of a 58-year-old woman applying to our emergency service for palpitation that was lasting for approximately 40 minutes that she was treated for 2 years with an oral medicine containing Diltiazem $\mathrm{HCl} 120$ mg $2 \times 1$. The patient indicated that she had no other disease and that she was using the medicine regularly; the following values have been obtained: brachial arterial pressure: 135/80 $\mathrm{mmHg}$, pulse: $210 / \mathrm{min}$, rhythmic-regular, $\mathrm{sPO}_{2}: 95 \%$, axillar fever: $36.7^{\circ} \mathrm{C}$. Her electrocardiography (ECG) has been determined as QRS with narrow tachycardia (Fig. 1). No acute ischemic modification has been observed. The blood electrolyte levels and Creatinine Kinase, Creatinine Kinase$\mathrm{MB}$ and Troponine-I levels used as cardiac indicators are considered normal. As the medicine used is Diltiazem $\mathrm{HCl}$, the first intervention was an intravenous $0.25 \mathrm{mg} / \mathrm{kg}$ dose. 
While the haemodynamic observations of the patient were normal, a $2^{\text {nd }}$ dose of $0.35 \mathrm{mg} / \mathrm{kg}$ after 15 minutes and a $3 \mathrm{rd}$ dose of Diltiazem $\mathrm{HCl} 0.35 \mathrm{mg} / \mathrm{kg}$ after another 15 minutes was administrated intravenously because the rate did not decrease in the monitored follow-up. After the third diltiazem dose, the cardiac rate became: 120/minute. Delta waves have been observed in the control ECG (Fig. 2). The patient was transferred to the coronary intensive care of the cardiology clinic for monitoring and treatment with WPW syndrome prediagnosis. Her echocardiography was normal. She was assigned to electrophysiological (EPS) study. During EPS, orthodromic tachycardia was induced again. An accessory pathway was found at right posterior localization based on intracardiac findings (Fig. 3), and successful ablation was achieved in the same session (Fig. 4). The patient for which a normal sinus rhythm is provided has been discharged with advices.

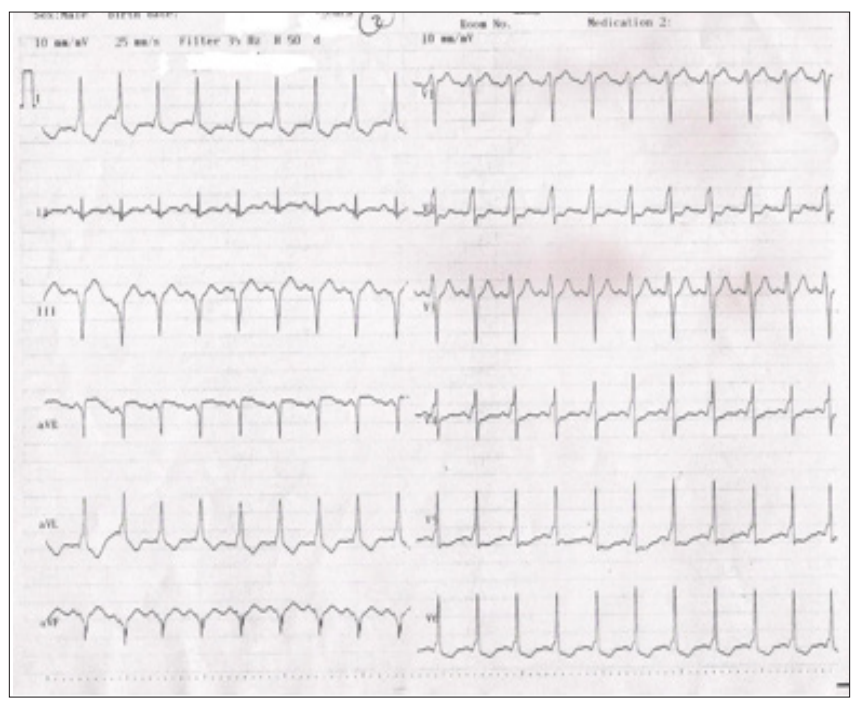

Fig. 1. Tachycardia with narrow QRS

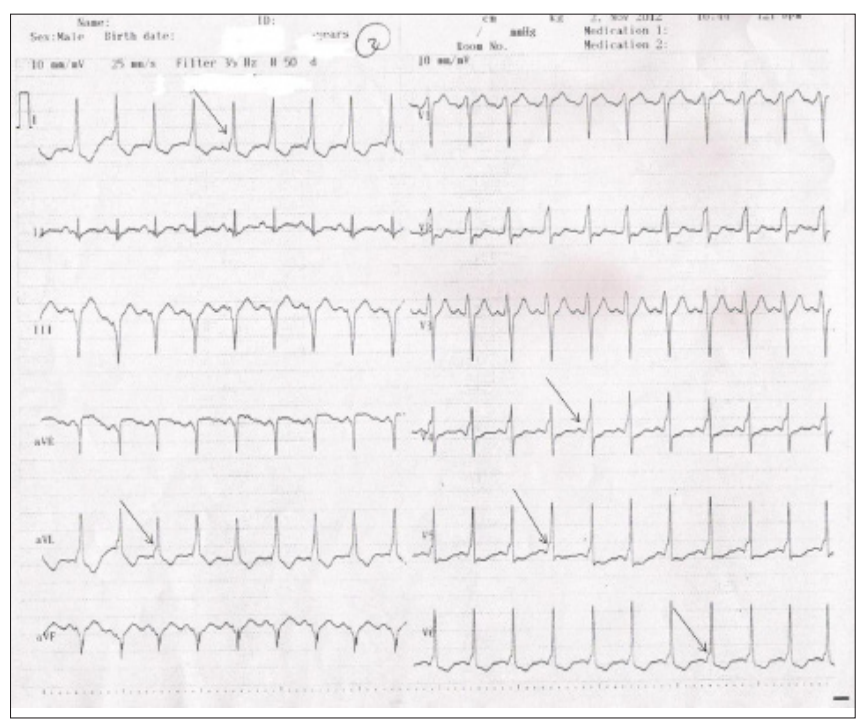

Fig. 2. Delta waves have been observed in the control ECG

\section{Discussion}

The accessory atrioventricular (AV) pathway that bypasses the AV node is the WPW syndrome. The reported incidence of WPW syndrome in the general population is $0.9 \%$ to $3 \%$ (Rosner et al., 1999). The ventricular myocardium is activated earlier than expected by an accessory conduction pathway

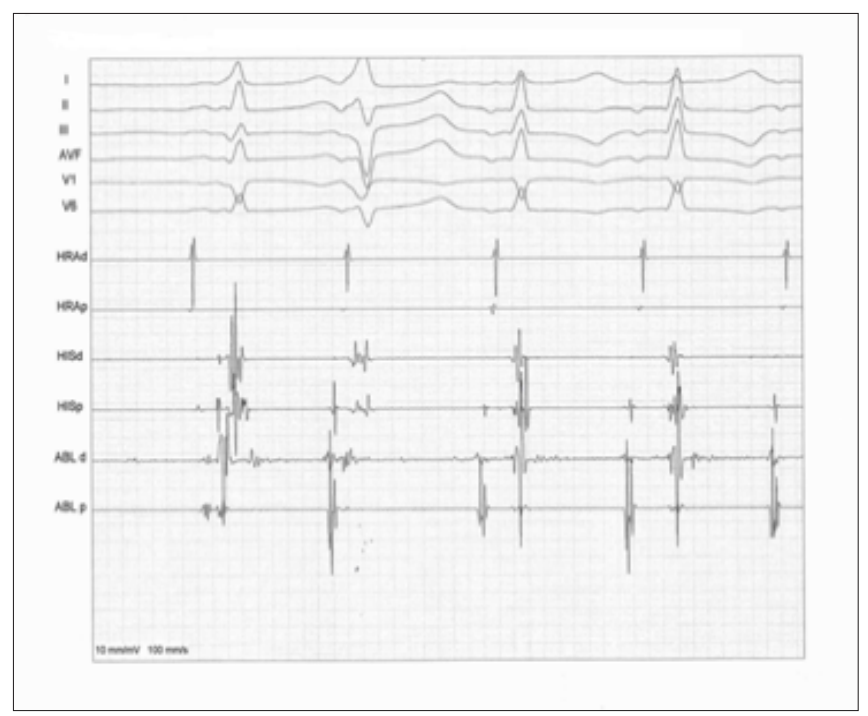

Fig. 3. An accessory pathway was found at right posteroseptal localization (parahisian) based on intracardiac findings, and successful ablation was achieved in the same figure.

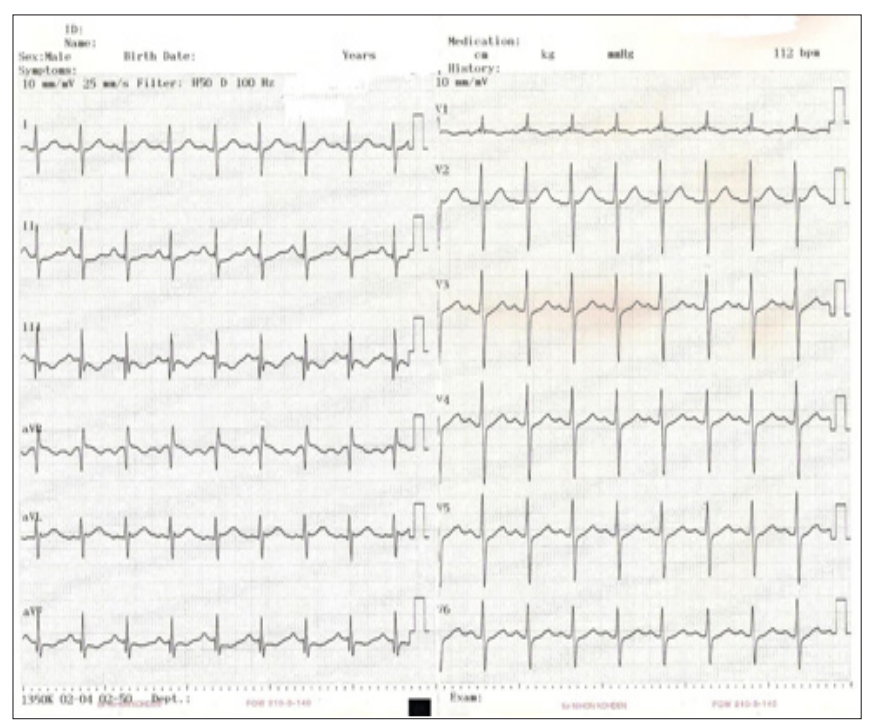

Fig. 4. Successful ablation

that allows a direct electrical connection between the atria and ventricles. In this way short PR interval and a delta wave are observed on ECG. AV re-entrant tachycardia and atrial fibrillation are observed (Bennett et al., 2012). Although many patients just present the symptoms throughout their life, approximately half of the patients with WPW syndrome experience symptoms secondary to tachyarrhythmias, such as paroxysmal supraventricular tachycardia, atrial fibrillation, atrial flutter, and, rarely, ventricular fibrillation and sudden death. The symptoms include palpitations, dizziness, syncope, and dyspnea. The diagnosis of WPW syndrome is usually made by electrocardiogram findings, but further testing may be warranted to confirm the diagnosis (Valderrama et al., 2004; Erdem et al., 2010). The most frequently encountered tachycardia in WPW syndrome is the reentrant tachycardia. The degeneration of reciprocating tachycardia to atrial fibrillation is not uncommon (Sung et al., 1977). In 95\% of WPW patients, there is paroxysmal AV reciprocating tachycardia (AVRT) with an antegrade conduction through the $\mathrm{AV}$ node and a retrograde conduction through the accessory pathway (orthodromic AVRT) producing a narrow QRS tachycardia (Mark et al., 2009). Amiodarone is 
frequently used for the treatment WPW syndrome caused by SVT control in this development (Bieganowska et al., 2006).

WPW syndrome patients had a high probability of developing PSVT and detailed ECG and electrophysiological examinations are required for early detection of PSVT and WPW syndrome (Ho et al., 2012). In patients with narrow QRS AVRT (WPW history not known or undiagnosed), the AV nodal blocker adenosine (or verapamil or diltiazem) is the most preferred drug, which interrupts the reentrant cycle by blocking the conduction through the AV node. The administration of adenosine for patients presenting wide QRS tachycardia as a diagnostic test should be discouraged as there is occurrence of ventricular fibrillation when used indiscriminately (Rozanski et al., 1982).

Nowadays, mostly beta blockers or $\mathrm{Ca}^{+2}$ channel blockers are preferred to moderate the rhythm for patients who present SVT in the ECG. However, when only ECG are used as a basis for the beginning of the treatment for diseases such as WPW syndrome that possesses some accessory pathways that are close to the normal conduction pathway, unexpected side effects may appear. Even if it is not widely used, the electrophysiological studies applied in important centers are really helpful. Catheter ablation is suggested for patients resuscitated from VF or for patients at high risk for clinical atrial fibrillation with a rapid ventricular response (Zips et al., 1995). As in our case, the ECG is used as a basis for Narrow QRS AVNRT to decrease the rhythmic AVNRT rate, and Diltiazem $\mathrm{HCl}$ treatment was applied and a WPW compatible rhythmic return ECG has been observed.

As a result, as accessory pathway diseases, being wrongly considered and treated as non complex SVT may lead to fatal arrhythmia by treatment, we tried to emphasize on the necessity to orientate the patients to the centers performing electrophysiological studies.

\section{REFERENCES}

Bennett, D.H., 2012. Wolff-Parkinson-White Syndrome, in Bennett's Cardiac Arrhythmias: Practical notes on interpretation and treatment, eighth edition, John Wiley \& Sons, Ltd, Oxford. doi: 10.1002/9781118432389.ch10.

Bieganowska, K, Rekawek, J, , Szumowski, L., Szymaniak, E., Brzezińska-Paszke, M., Miszczak-Knecht M., Glinka-Kazimierska, E., Kawalec, W., Nowak I., Walczak, F., 2006. Morgagni-Adams-Stokes after adenosine injection in a patient with WPW syndrome-a case report. Kardiol. Pol. 64, 1453-1457.

Erdem, A., Madak, N., Yilmaz, A., Yontar, O.C., Yucel, H., Gul, I., Tandogan, I., 2010. Development of malignant ventricular arrhythmias in a young male with WPW Pattern. Indian Pacing and Electrophysiology J. 10, 195-200.

Ho, H.H., Yeh. S.J., Tsai, W.P., Wang. C.M., Chen. J.Y., 2012. Paroxysmal supraventricular tachycardia and Wolff-Parkinson-White syndrome in ankylosing spondylitis: A large cohort observation study and literature review. Semin Arthritis Rheum. 42, 246-253.

Mark, D.G., Brady, W.J., Pines J.M., 2009. Preexcitation syndromes: diagnostic consideration in the ED. Am. J. Emerg. Med. 27 , 878-888.

Rosner, M.H., Brady, W.J.Jr., Kefer, M.P., Brady, W.J., Martin, M.L., 1999. Electrocardiography in the patient with the Wolff-Parkinson-White syndrome: diagnostic and initial therapeutic issues. Am. J. Emerg. Med. 17, 705-714.

Rozanski, J.J., Zaman, L., Castellanos, A., 1982. Electrophysiologic effects of diltiazem hydrochloride on supraventricular tachycardia. Am. J. Cardiol. 49, 621-628.

Sung, R.J., Castellanos, D., Mallon, S.M., Bloom, M.G., Gelband, H., Myerburg, R.J., 1977. Mechanisms of spontaneous alteration between reciprocating tachycardia and atrial flutter fibrilation in the Wolff- parkinson-White syndrome. Circulation. 56, 409.

Valderrama, A.L., 2004 Wolff-Parkinson-White syndrome: essentials for the primary care nurse practitioner. J. Am. Acad. Nurse. Pract. 16, 378-383.

Zipes, D.P., DiMarco, J.P., Gillette, P.C., Jackman, W.M., Myerburg, R.J., Rahimtoola, S.H., Ritchie J.L., Cheitlin, M.D., Garson, A. Jr., Gibbons, R.J., P.C., 1995. Guidelines for clinical intracardiac electrophysiological and catheter ablation procedures. A report of the American College of Cardiology/American Heart Association Task Force on Practice Guidelines (Committee on Clinical Intracardiac Electrophysiologic and Catheter Ablation Procedures), developed in collaboration with the North American Society of Pacing and Electrophysiology. J. Am. Coll Cardiol. 26, 555-573. 